\title{
Imaging of Individual Vacancies Using Electron Channeling Contrast in STEM
}

\author{
Jared M Johnson and Jinwoo Hwang
}

Department of Materials Science and Engineering, The Ohio State University, Columbus, OH 43212

Low-angle annular dark field (LAADF) imaging in scanning transmission electron microscopy (STEM) is known to be highly sensitive to the structural defects in the material, including point defects and dislocations, as such defects may lead to de-channeling of the electron beam [1]. Using multislice STEM simulation, we show that, by simultaneously using multiple narrowly-selected LAADF angle ranges, such as 20-30 and 30-40 mrad, the image intensity of the vacancy-containing column can become highly dependent to the depth of the vacancy along the beam direction. The result implies that the method can potentially be used to measure the 3 -dimensional positions of individual vacancies.

Our multislice simulation has revealed that, when the vacancy is present in the atomic column of a crystal $\left(\beta-\mathrm{Ga}_{2} \mathrm{O}_{3}\right)$, it creates extra "ripples" at the angle range between $\sim 20$ and 40 mrad in reciprocal space (Fig. 1). Such ripple is still present when phonon vibration is introduced into the simulation. More importantly, the ripple changes its shape (both amplitude and phase) as a function of the depth position of the vacancy. This implies that, if the ADF detector is placed in that angle range, the intensity of the vacancy-containing column can become sensitive to the depth position of the vacancy. For example, using 20-30 mrad angle, the intensity of the vacancy-containing column can decrease up to $\sim 20 \%$ of the intensity of the column with no vacancy (Fig. 2a). However, when a different angle range, 30-40 mrad, was used, the profile of the intensity dependence on the vacancy depth is significantly different from the 20-30 mrad case (Fig. 2b). Therefore, using 20-30 and 30-40 mrad angle ranges simultaneously could provide the depth position of the vacancy with very high accuracy. We also found that, this channeling contrast imaging method can be used for samples up to $\sim 6 \mathrm{~nm}$ depending on the crystal orientation, and also work even if there is small atomic displacement nearby the vacancy [2].

Experimental realization of the proposed imaging mode will require overcoming several obstacles. While the change in the column intensity by the vacancy is rather significant (Fig. 2a and b), the precision of the vacancy depth position will ultimately be limited by experimental uncertainties, such as non-ideal surface conditions, sample drift, and Poisson noise. We estimate that the experimental uncertainties needs to be kept lower than $\sim 5 \%$ of the column intensity in order to realize this method. In addition, at the moment it remains unclear how to distinguish the de-channeling signal from point defects from that of other potential defects. For example, LAADF is also known to be sensitive to surface features, but a more quantitative understanding on the effect of surface features to the LAADF signal may be required. Channeling may also occur from extended defects or the strain in the material.

More importantly, the need of multiple, narrowly selected detector geometry in STEM adds further challenge. While choosing $\sim 20-30 \mathrm{mrad}$ ADF range is possible in our STEM (FEI Titan), the outer angle range of the second ADF detector may need to be limited using an objective aperture to realize the 30-40 mrad detection range. ADF detectors also have other issues, including non-uniformity in gain [3], which can increase uncertainty in the quantitative analysis [4]. An alternative, and probably the better method is to use the newly developed pixelated STEM detector [5], which has demonstrated the full-field scanning diffraction imaging at the atomic scale with high dynamic range and signal to noise ratio. 


\section{References}

[1] D. A. Muller, N. Nakagawa, A. Ohtomo, J. L. Grazul, H.Y. Hwang, Nature 430, 657 (2004).

[2] J. Johnson, S. Im, W. Windl, J. Hwang, Ultramicroscopy 172, 17 (2017).

[3] S. D. Findlay and J. M. LeBeau. Ultramicroscopy, 124, 52 (2013).

[4] J. Hwang et al., Physical Review Letters 111, 266101 (2013).

[5] M. W. Tate, et al., Microsc. Microanal. 22, 237 (2016).
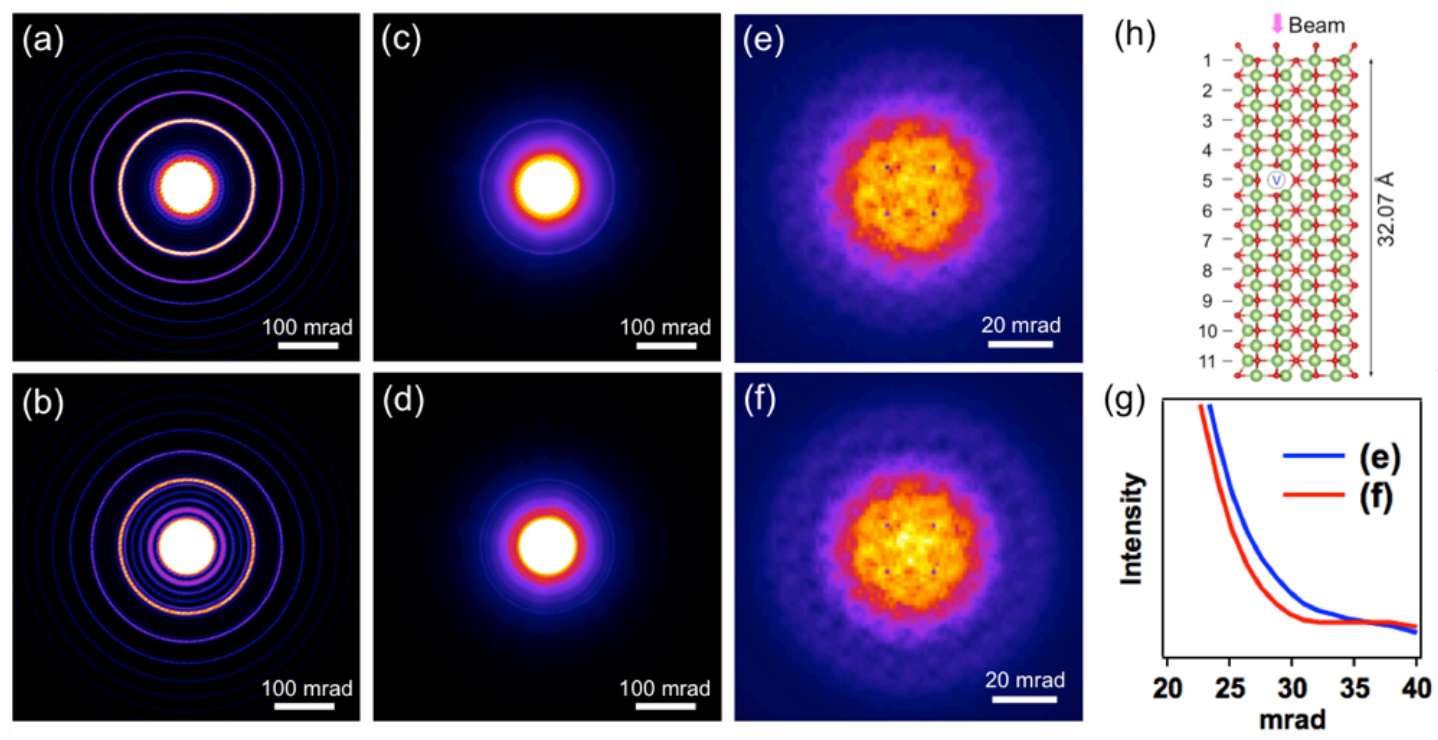

Figure 1. CBED simulated with a probe at the Ga column with (a) no vacancy and (b) a vacancy at the $6^{\text {th }}$ position shown in (h) without thermal diffuse scattering (TDS). (c and d) Same simulations but with TDS for (a) and (b) cases, respectively. (e and f) Zoomed-in versions of (c) and (d), respectively, showing the intensities at low angles. (a) to (d) are on a linear scale with the intensity saturated at the center to make the high angle scattering more visible. (e) and (f) are presented with $\gamma=0.5$. (g) Annular averaged intensities of the patterns in (e) and (f) to show the differences between them on a linear scale. (h) Schematic of $\beta-\mathrm{Ga}_{2} \mathrm{O}_{3}$ in the simulation along the $[010]_{\mathrm{m}}$ beam direction. Probe convergence angle $=$ $9.6 \mathrm{mrad}$, and sample thickness $=33 \AA$.
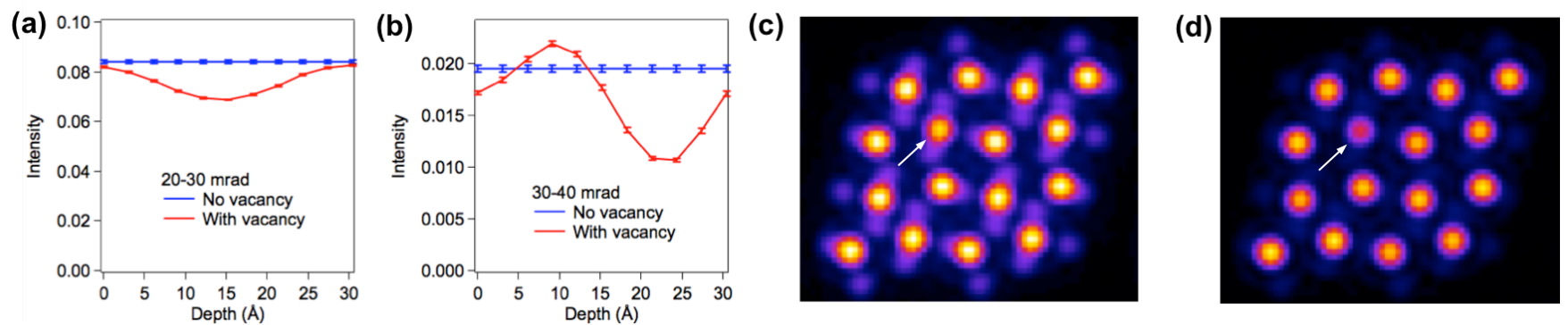

Figure 2. Column intensity as a function of Ga vacancy depth position (red curve) compared to the column intensity without a vacancy (blue curve) for detection angles (a) 20-30 mrad, (b) 30-40 mrad, along $[010]_{\mathrm{m}}$ of $\beta-\mathrm{Ga}_{2} \mathrm{O}_{3}$. (c) and (d) are simulated images with a vacancy placed at $15 \AA$ in depth with a 20-30 mrad detector and at $25 \AA$ with a 30-40 mrad detector, respectively. All simulations included TDS, a probe convergence half angle of $9.6 \mathrm{mrad}$, and sample thickness $=33 \AA$. 